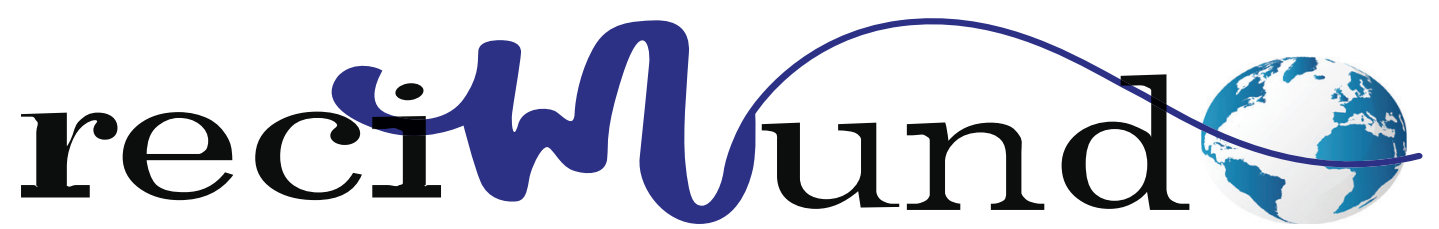

Revista Científica Mundo de la Investigación y el Conocimiento

DOI: 10.26820/recimundo/4.(4).noviembre.2020.228-235

URL: http://recimundo.com/index.php/es/article/view/942

EDITORIAL: Saberes del Conocimiento

REVISTA: RECIMUNDO

ISSN: 2588-073X

TIPO DE INVESTIGACIÓN: Artículo de revisión

Código UNESCO: 32 Ciencias Médicas

PAGINAS: $228-235$

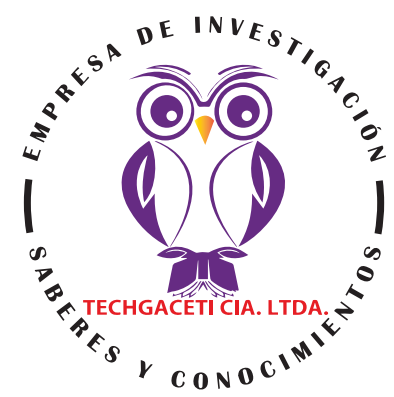

\title{
Una mirada distinta al embarazo adolescente
}

A different view of teen pregnancy

Uma visão diferente da gravidez na adolescência

Dra. Ruth Elizabeth Maldonado Rengel', PhD Rosario Suarez²,

Dra. Ana Lizette Rojas Rodríguez ${ }^{3}$,Dra. Yadira Gavilanes Cueva ${ }^{4}$

RECIBIDO: 31/08/2020 ACEPTADO: 02/09/2020 PUBLICADO: 10/11/2020

1. Magister en Hebeatría; Doctora en Medicina y Cirugía; Docente Investigadora de la Universidad Técnica Particular de Loja, Docente del Departamento de Ciencias de la Salud en la carrera de medicina en pregrado y postgrado; Loja, Ecuador; remaldonado6@utpl.edu.ec; iD https://orcid.org/0000-0002-6435-1729

2. Pdh en Inmunología: Inflamación, Enfermedades del Sistema Inmune y Nuevas Terapias; Docente de pregrado y postgrado en Medicina Interna en la Universidad Técnica Particular de Loja; Loja, Ecuador; rsuarez2@utpl.edu.ec; iD https://orcid. org/0000-0003-0419-030X

3. Especialista en Pediatría; Médico; Docente en la Universidad Técnica Particular de Loja en la titulación de Medicina con los componentes de Nutrición, Laboratorio de Histología, Primeros Auxilios, Nutrición, Familia y Vida 1,1, Pediatría, Ética Médica; Docente en el Postgrado de la Especialidad en Medicina Familiar del Componente Niño; Médico tratante de Pediatría en el Hospital UTPL; alrojas4@utpl.edu.ec; (iD https://orcid.org/0000-0002-5708-8782

CORRESPONDENCIA

Ruth Elizabeth Maldonado Rengel

rsuarez2@utpl.edu.ec

Loja, Ecuador 


\section{RESUMEN}

Introducción: El embarazo adolescente (EA), es considerado un problema de salud pública respecto del mayor riesgo de complicaciones maternas, perinatales y para el desarrollo psicosocial de la adolescente, evidenciándose altas tasas de este en Ecuador. Objetivo: Determinar los factores de riesgo para EA en adolescentes escolarizadas de la ciudad de Loja, Ecuador. Material y Métodos: Se estudiaron 632 adolescentes mujeres, estudiantes de instituciones de educación media de Loja, Ecuador (enero 2017- enero 2018). Los factores de riesgo para EA estudiados fueron: edad de inicio de vida sexual, violencia intrafamiliar o en la pareja, creencia en mitos sobre sexualidad y ser hija de madre adolescente. Se realizó un muestreo no probabilístico por conveniencia, y se aplicó una encuesta semiestructurada con preguntas dicotómicas sobre los factores de riesgo para EA, previo a lo cual se pidió el consentimiento informado de los representantes y asentimiento informado de las adolescentes. Resultados: La frecuencia total de EA fue de 3,6 \%. Los factores de riesgo para embarazo adolescente determinados en este estudio fueron ser hija de madre adolescente (HMA) $(\mathrm{OR}=8,12$; IC95 \% 2,51-26,24) y la violencia de pareja (VP) (OR=6,11; IC95\% 1,12-37,04). Conclusiones: La violencia en la pareja y el ser hija de madre adolescente son los factores de riesgo para embarazo adolescente identificados en este estudio.

Palabras clave: embarazo, adolescente.

\section{ABSTRACT}

Introduction: Adolescent pregnancy (AP), which has high rates in Ecuador, is considered a public health problem related to the increased risk of maternal and perinatal complications and the psychosocial development of adolescents Objective: To determine the risk factors for AP in adolescents in the city of Loja, Ecuador. Material and Methods: 632 teenage female students from high school institutions in Loja, Ecuador (January 2017-January 2018) were studied. The risk factors for AP studied were age of initiation of sexual life, domestic or partner violence, belief in myths about sexuality, and being the daughter of an adolescent mother. A non-probabilistic convenience sampling was carried out, and a semi-structured survey was applied with dichotomous questions about the risk factors for AP, prior to which the informed consent of the representatives and informed assent of the adolescents was requested. Results: The total frequency of AP was 3.6\%. The risk factors for adolescent pregnancy determined in this study were being the daughter of an adolescent mother (AMF) $(\mathrm{OR}=8.12 ; 95 \% \mathrm{Cl} 2.51-26.24)$ and intimate partner violence (VP) $(\mathrm{OR}=6,11 ; 95 \% \mathrm{Cl}$ 1.12-37.04). Conclusions: Intimate partner violence and being the daughter of an adolescent mother are the risk factors for adolescent pregnancy identified in this study.

Keywords: pregnancy, adolescent.

\section{RESUMO}

Introdução: A gravidez na adolescência (PA), que apresenta altas taxas no Equador, é considerada um problema de saúde pública relacionado ao aumento do risco de complicações maternas e perinatais e ao desenvolvimento psicossocial das adolescentes. Objetivo: Determinar os fatores de risco para PA em adolescentes na cidade de Loja, Equador. Material e métodos: foram estudadas 632 alunas adolescentes de instituições de ensino médio em Loja, Equador (janeiro de 2017 a janeiro de 2018). Os fatores de risco para PA estudados foram idade de início da vida sexual, violência doméstica ou conjugal, crença em mitos sobre a sexualidade e ser filha de mãe adolescente. Realizou-se amostragem não probabilística por conveniência e aplicou-se questionário semiestruturado com questões dicotômicas sobre os fatores de risco para PA, antes da solicitação do consentimento informado dos representantes e consentimento informado dos adolescentes. Resultados: A frequência total de PA foi de 3,6\%. Os fatores de risco para gravidez na adolescência determinados neste estudo foram ser filha de mãe adolescente (AMF) (OR = 8,12; IC95\% 2,51-26,24) e violência por parceiro íntimo (VP) (OR = 6, 11; IC95\% 1.12-37.04). Conclusões: Violência por parceiro íntimo e ser filha de mãe adolescente são os fatores de risco para gravidez na adolescência identificados neste estudo.

Palavras-chave: gravidez, adolescente. 


\section{Introducción}

La adolescencia está definida por la Organización Mundial de la Salud (OMS) como la etapa comprendida entre los 10 y 19 años de edad (1); la adolescencia temprana va entre los 10-14 años y la tardía entre los 1519 años. Durante esta etapa, se producen cambios a nivel físico como el crecimiento puberal y el desarrollo de los caracteres sexuales secundarios, así como, la adquisición de nuevas habilidades sociales, cambios cerebrales que mejoran su nivel de cognición y cambios psicológicos que lo conducen a la formación de su identidad adulta. (2).

El embarazo adolescente se define como aquel que ocurre durante el período de la adolescencia o cuando aún se tiene dependencia familiar independiente de su edad ginecológica.

En Ecuador, en el año 2014, 38,74 \% de los nacidos vivos fueron de madres adolescentes (3). La tasa global de fecundidad en Ecuador es de 2,2 (4). Ecuador es el tercer país en la región con la tasa más alta de embarazos adolescentes detrás de Nicaragua y República Dominicana.(5)

El EA es considerado por la OMS como un problema de salud pública puesto que implica un mayor riesgo de complicaciones para la madre como es el caso de hemorragia post parto, parto prolongado, anemia, complicaciones por aborto, así como complicaciones psicosociales como es el caso del fracaso en la adquisición de la independencia, en el logro de su identidad, en la continuidad de sus estudios, alteración de su proyecto de vida, etc., $(6,7)$

Además, el EA es un factor reconocido de riesgo de morbilidad y mortalidad materna, perinatal e infantil debido a la mayor posibilidad de presentar bajo peso al nacer, prematurez y retardo del crecimiento intrauterino, además de mayor tendencia al destete precoz, a presentar diarreas e infecciones respiratorias agudas $(8,9)$.

Para poder prevenir estos embarazos es necesario conocer cuáles son los factores de riesgo que anteceden su presentación y luego organizar programas de salud pública que los modifiquen. En Ecuador se han publicado algunos estudios que han permitido conocer los posibles factores de riesgo que tienen asociación significativa con el EA en adolescentes de Guayaquil y Orellana $(10,11)$. Los factores de riesgo detectados fueron: inicio temprano de la actividad sexual, falta de conocimientos sobre salud reproductiva y falta de estructura familiar estable (11). En otro estudio se reportó factores como: inicio temprano de la actividad sexual, falta de uso de contraceptivos, residencia en vivienda muy pobre, haber sufrido de abuso sexual previo y falta de estructura familiar estable (12)

Por lo antes mencionado, así como las implicaciones para la salud de la madre y su hijo se consideró la realización de este estudio cuyo objetivo fue: Determinar los factores de riesgo para EA en adolescentes escolarizadas de la ciudad de Loja, Ecuador.

\section{Materiales y métodos}

Se realizó un estudio descriptivo sobre factores de riesgo para EA en instituciones de educación media de la ciudad de Loja Ecuador entre enero del 2017 y enero del 2018. Se realizó un muestreo no probabilístico por conveniencia, de 632 adolescentes mujeres escolarizadas de un universo de 30.252 distribuidas en 10 establecimientos de educación media de la ciudad de Loja. Los criterios de inclusión fueron: tener entre 10 y 19 años de edad, sexo femenino, ser estudiante regular de las instituciones participantes en el estudio, firmar el asentimiento informado y el consentimiento informado por sus padres o representantes. Los criterios de exclusión fueron: adolescentes impedidas de realizar la encuesta. 
La variable dependiente del estudio fue el EA (actual o previo) mientras que las variables independientes fueron: ser hija de madre adolescente, edad de inicio vida sexual, creencia de mitos falsos sobre anticoncepción, violencia intrafamiliar y violencia de pareja. Las respuestas posibles fueron dicotómicas, es decir, afirmativas o negativas. La encuesta fue aplicada por estudiantes de medicina de la Universidad Técnica Particular de Loja (UTPL) bajo la supervisión directa de los investigadores.

Las variables cuantitativas se resumieron con el promedio y la desviación estándar mientas que las variables cualitativas se hicieron con el número y porcentaje de sujetos en cada categoría. Los promedios se compararon con análisis de varianza de una vía y las proporciones con la prueba Chi cuadrado. La prevalencia de EA fue estimada y asociada con un intervalo de confianza de $95 \%(95 \%$ IC) .

La asociación entre cada uno de los factores de riesgo y la prevalencia de EA fue medida con el odds ratio (OR) con su 95\% IC respectivo. Una regresión logística permitió ajustar por estas variables confundentes previamente definidas. Se describió la presencia de los factores de riesgo en la muestra y se realizó la prueba de $f 2$ para compararlos entre las embarazadas y no embarazadas. Con regresión logística se calculó la influencia relativa de cada uno de ellos. Se fijó una $\mathrm{p}<0,05$ para determinar la significancia estadística. Los datos se pro- cesaron mediante el programa SPSS 20.0. Consideraciones éticas.

Los representantes, madre o padre o tutor legal, de todas las adolescentes incluidas firmaron un consentimiento informado en el que aprobaron la utilización de sus datos, con la garantía del anonimato. Las adolescentes también firmaron un asentimiento informado.

\section{Resultados}

Este estudio seleccionó 632 adolescentes de 10-19 años en 10 instituciones educativas de la ciudad de Loja, públicas (4), privadas (3) y cofinanciadas (3). La prevalencia total de EA fue 3,6\%. En las instituciones educativas cofinanciados la prevalencia de EA fue discretamente mayor $(5,7 \%)$ y no hubo embarazos en las de tipo privado.

La mayoría de embarazos ocurrieron en adolescentes mayores (95,7\%). La violencia intrafamiliar fue más frecuente en adolescentes de colegios cofinanciados que en públicos y privados $(28,1 \%$ vs $27,4 \%$ y $13,3 \%$, respectivamente). La violencia de pareja en cambio, fue más alta en públicos (4,4\% vs $2,4 \%$ y $0,5 \%)$, al igual que el antecedente de ser hija de madre adolescente (públicos 46,2\% vs 39,5\% en cofinanciados y $21 \%$ en privados) y el haber iniciado la vida sexual (25,9\% en públicos, $24,3 \%$ los cofinanciados y $9 \%$ los privados). 
MALDONADO RENGEL, R. E., SUAREZ, R., \& ROJAS RODRÍGUEZ, A. L.

Tabla 1. Factores de Riesgo para Embarazo adolescente Loja-Ecuador

\begin{tabular}{|c|c|c|c|c|}
\hline \multirow{2}{*}{\multicolumn{2}{|c|}{ Factor de Riesgo }} & \multicolumn{2}{|c|}{ Embarazo Adolescente } & \multirow{3}{*}{ valor $\mathbf{P}$} \\
\hline & & \multirow{2}{*}{$\begin{array}{l}\text { Si \% } \\
22,95 \\
\end{array}$} & \multirow{2}{*}{$\frac{\text { NO } \%}{61,9}$} & \\
\hline & $\begin{array}{l}\text { Adolescencia } \\
\text { mayor }\end{array}$ & & & \\
\hline \multirow{4}{*}{$\begin{array}{l}\text { Edad de inicio de } \\
\text { vida sexual }\end{array}$} & 11 a 12 años & 4,5 & 2,0 & \multirow{4}{*}{0,03} \\
\hline & 13 a 14 años & 4,5 & 22,8 & \\
\hline & 15 a 16años & 59,1 & 63,4 & \\
\hline & 17 a 19 años & 31,9 & 11,9 & \\
\hline \multicolumn{5}{|l|}{ Creencia en mitos } \\
\hline \multirow{2}{*}{$\begin{array}{l}\text { Mito 1: creer que la } \\
\text { primera vez que se } \\
\text { tienen relaciones } \\
\text { sexuales no hay } \\
\text { riesgo de emba- } \\
\text { razo }\end{array}$} & Cree en el mito & 26,1 & 28,9 & \multirow[b]{2}{*}{0,73} \\
\hline & No Cree en el mito & 73,9 & 70,0 & \\
\hline \multirow{2}{*}{$\begin{array}{l}\text { Mito 2: si se eyacu- } \\
\text { la fuera no hay ries- } \\
\text { go de embarazo }\end{array}$} & Cree en el mito & 73,9 & 61,1 & \multirow{2}{*}{0,35} \\
\hline & No Cree en el mito & 26,1 & 37,4 & \\
\hline \multirow{2}{*}{$\begin{array}{l}\text { Mito 3: tomar } \\
\text { anticonceptivos } \\
\text { aumenta el peso } \\
\text { corporal }\end{array}$} & Cree en el mito & 52,2 & 45,5 & \multirow[b]{2}{*}{0,26} \\
\hline & No Cree en el mito & 39,1 & 51,7 & \\
\hline \multirow{2}{*}{$\begin{array}{l}\text { Mito 4: tomar } \\
\text { anticonceptivos } \\
\text { produce cáncer }\end{array}$} & Cree en el mito & 17,4 & 33,3 & \multirow{2}{*}{0,13} \\
\hline & No Cree en el mito & 73,9 & 63,7 & \\
\hline \multirow{2}{*}{$\begin{array}{l}\text { Mito 5: usar condón } \\
\text { produce alergias }\end{array}$} & Cree en el mito & 60,9 & 62,1 & \multirow{2}{*}{0,48} \\
\hline & No Cree en el mito & 30,4 & 34,6 & \\
\hline \multirow{2}{*}{$\begin{array}{l}\text { Mito 6: en la prime- } \\
\text { ra relación no es } \\
\text { necesario ningún }\end{array}$} & Cree en el mito & 13,0 & 9,5 & \multirow{2}{*}{0,5} \\
\hline & No Cree en el mito & 87,0 & 88,0 & \\
\hline \multirow{2}{*}{$\begin{array}{l}\text { Mito 7: tomar ducha } \\
\text { vaginal u orinar } \\
\text { después de rela- } \\
\text { ciones sexuales } \\
\text { previene embarazo }\end{array}$} & Cree en el mito & 47,8 & 32,0 & \multirow[b]{2}{*}{0,28} \\
\hline & No Cree en el mito & 47,8 & 64,2 & \\
\hline \multirow{2}{*}{$\begin{array}{l}\text { Violencia Intrafa- } \\
\text { miliar }\end{array}$} & $\begin{array}{l}\text { Presencia de vio- } \\
\text { lencia Intrafamiliar }\end{array}$ & 39,1 & 22,3 & \multirow{2}{*}{0,06} \\
\hline & $\begin{array}{l}\text { Ausencia de Violen- } \\
\text { cia intrafamiliar }\end{array}$ & 60,9 & 77,7 & \\
\hline \multirow{2}{*}{ Violencia de pareja } & $\begin{array}{l}\text { Presencia de Vio- } \\
\text { lencia de pareja }\end{array}$ & 13,0 & 2,0 & \multirow{2}{*}{0,01} \\
\hline & $\begin{array}{l}\text { Ausencia de Violen- } \\
\text { cia de pareja }\end{array}$ & 87,0 & 98,0 & \\
\hline \multirow{2}{*}{$\begin{array}{l}\text { Antecedente de } \\
\text { madre adolescente }\end{array}$} & $\begin{array}{l}\text { Antecedente pre- } \\
\text { sente }\end{array}$ & 73,9 & 34,2 & \multirow{2}{*}{$<0,001$} \\
\hline & $\begin{array}{l}\text { Antecedente au- } \\
\text { sente }\end{array}$ & 26,1 & 65,8 & \\
\hline
\end{tabular}

Autores: Investigadores.

Fuente: base de datos 
Tabla 2. Resultados de regresión logística de los factores de riesgo asociados con Embarazo Adolescente

\begin{tabular}{|l|l|l|l|l|}
\hline & \multirow{2}{*}{ OR } & \multicolumn{2}{|l|}{ IC 95\% } & \\
\cline { 3 - 5 } & & inferior & superior & \\
\hline $\begin{array}{l}\text { Antecedente de } \\
\text { madre adolescente }\end{array}$ & 8,12 & 2,51 & 26,24 & $<0,001$ \\
\hline Violencia de pareja & 6,11 & 1,12 & 37,04 & 0,03 \\
\hline
\end{tabular}

Autores: Investigadores.

Fuente: base de datos

Se encontró asociación significativa entre EA el ser hija de madre adolescente $(p<0,001), \quad$ y la presencia de violencia de pareja $(p=0,01)$ La creencia en mitos erróneos o falsos sobre sexualidad ( la primera vez que se tienen relaciones sexuales no hay riesgo de embarazo, si se eyacula fuera no hay riesgo de embarazo, tomar anticonceptivos aumenta el peso corporal, tomar anticonceptivos produce cáncer, usar condón produce alergias, en la primera relación no es necesario ningún método anticonceptivo y tomar ducha vaginal u orinar después de relaciones sexuales previene embarazo) no tuvieron relación con el embarazo en adolescentes.

Por regresión logística se determinó como factores de riesgo del EA, el hecho de ser hija de madre adolescente y la presencia de violencia de pareja. El factor de riesgo más fuertemente asociado al embarazo fue ser hija de madre adolescente $(\mathrm{OR}=8,12$; IC95\% 2,51-26,24) y tercero la presencia de violencia de pareja $(\mathrm{OR}=6,11$; IC95\% 1,1237,04).

\section{Discusión}

Respecto de los factores de riesgo para embarazo adolescente como es la violencia de pareja estudios realizados por Baeza y col indicaron que pese a que encontraron violencia familiar presente en el 22,9\% de las adolescentes estudiadas, este no fue un factor de riesgo para EA, esto puede deberse al hecho que la violencia de pareja en el adolescente a diferencia de la violencia intrafamiliar conlleva a la manipulación sobre el hecho de mantener relaciones sexuales, así como las amenazas de abandono que la pareja le confiere, lo cual en la etapa de la adolescencia donde aún su personalidad no se ha formado y se carece de la madurez emocional e intelectual para poner límites o incorporar medidas de prevención ante el EA, facilitan que este hecho ocurra. $(13,14,15,1)$. Con lo manifestado concuerdan los estudios realizados por Svanemyr et al., y Sezgin et al., que encontraron que la violencia de pareja era más común entre aquellas mujeres que se embarazan en la adolescencia $(16,17)$, lo cual pese a ser estudios en entornos sociales diferentes implican una realidad en común que es el hecho que en una relación de "poder" en la pareja, las adolescentes son obligadas o inducidas a tener relaciones sexuales y por ende a EA, sumado a además a las creencias de "amor romántico" propias de esta época de la vida. (18)

Así mismo, otro de los factores de riesgo implicados en el riesgo de EA en nuestro estudio fue el ser hija de madre adolescente, este hecho concuerda con lo presentado en algunos estudios que muestran que las hijas de madres adolescentes tienen alrededor de dos veces más riesgo de llegar a ser madres adolescentes y además se embarazan incluso a edades más tempranas que sus propias madres $(18,19,20)$. Esto puede deberse a la repetición de conductas permisivas en la crianza de las adoles-

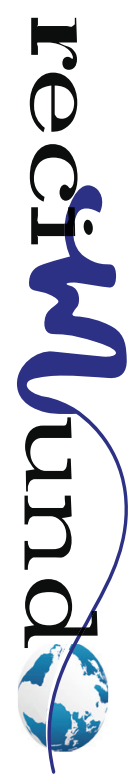


centes que facilitan el inicio precoz de vida sexual, a la repetición de patrones de relacionamiento, que permiten que las adolescentes al igual que sus madres se embaracen en este período de vida.

Estos dos factores relacionados con la presencia de embarazo adolescente en nuestro estudio responden a la organización social de la sociedad ecuatoriana en donde la violencia de pareja se presenta en el $43 \%$ de los casos, de los cuales el 40,8 $\%$ corresponde a violencia psicológica y el $25 \%$ violencia física, que implica como lo hemos explicado antes la manipulación, coartación y la amenaza de abandono que implican un mayor riesgo de un embarazo adolescente. (21). Así como a rol pasivo que muchas mujeres aún mantienen en la sociedad ecuatoriana, donde se considera a la mujer como objetos sexuales, no se les permite opinar abiertamente, donde todavía son juzgadas por la conductas que toman especialmente respecto de la libertad sobre el ejercicio adecuado de su sexualidad, se limita o manipula la información sobre salud sexual y reproductiva, y se sigue pensando que el rol más importante de la mujer en la sociedad es la maternidad, situaciones todas que constituyen en el terreno fértil para que el EA siga teniendo las altas tasa que actualmente tiene el país.

\section{Conclusiones}

El ser hija de madre adolescente, y la violencia de pareja constituyeron los principales factores de riesgo para EA en este estudio.

\section{Bibliografía}

1. Organización Mundial de la Salud (OMS). El embarazo en la adolescencia. 2020. Disponible en: https://www.who.int/es/news-room/fact-sheets/detail/adolescent-pregnancy

2. Kliegman RM Tratado de Pediatría. Vol 1. 19 Ed. Elsevier: 2019

3. Instituto Nacional de Estadísticas y Censos, 2016. Ecuador. Compendio de estadísticas 2015 (pági- nas 82 y 83, para nacidos vivos totales y para nacidos vivos en adolescentes). Disponible en: http:// www.ecuadorencifras.gob.ec/compendio-estadistico-2015/ Consultado en: Julio 5, 2018.

4. Encuesta Nacional de Salud y Nutrición ENSANUT INEC 2019 disponible en la web: https://www.ecuadorencifras.gob.ec/documentos/web-inec/Estadisticas_Sociales/ENSANUT/ENSANUT_2018/ Principales\%20resultados\%20ENSANUT_2018. pdf

5. García A. Ecuador, tercer país en la región con la tasa más alta de embarazos entre 10 y 19 años. El Comercio. 2018. Disponible en la web: https:// www.elcomercio.com/actualidad/ecuador-estadisticas-embarazo-adolescente-mama.html.

6. Flores-Valencia, M. E., Nava-Chapa, G., \& Arenas-Monreal, L. Embarazo en la adolescencia en una región de México: un problema de Salud Pública. 2017. Revista de Salud Pública, 19, 374378.

7. Sanabria Negrín JG, Fernández-Montequín ZC. Factores de riesgo del embarazo en adolescentes y jóvenes. Bata, Guinea Ecuatorial. Rev Ciencias Médicas [Internet]. 2019 [citado: fecha de acceso]; 23(1): 119-134. Disponible en: http://revcmpinar.sld.cu/index.php/publicaciones/article/ view/3770

8. Bársena A. Embarazo adolecente, alerta temprana de la desigualdad. Columna de opinión de la Secretaria Ejecutiva de la CEPAL. [Online]. 2013 [cited 2016 Octubre 22. Available from: http:// www.cepal.org/es/articulos/embarazo-adolescente-alerta-temprana-de-la-desigualdad.

9. OPS/OMS. Las condiciones de salud en las Américas 2017. Disponible en: http://www.paho. org/salud-en-las-americas-2017/wp-content/ uploads/2017/09/Print-Version-Spanish.pdf Consultado en: Junio 28, 2018

10. Jeha D, Usta I, Ghulmiyyah L, Nassar A. A review of the risks and consequences of adolescent pregnancy. J Neonatal Perinatal Med. 2015; 8: 1-8.

11. Chedarui PA, Hidalgo LA, Chávez MJ, San Miguel G. Determinant factors in Ecuador related to pregnancy among adolescents aged 15 or less. J Perinat Med. 2004; 32: 337-341.

12. Goicolea I, Wulff M, Öhman A, San Sebastián M. Risk factors for pregnancy among adolescent girls in Ecuador's Amazon basin: a case-control study. Rev Panam Salud Pública/Pan Am J Public Health. 2009; 26; 3: 221-228.

13. Gómez-Mercado CA, Mejía-Sandoval G. Prevalencia de embarazo y características demo- 
gráficas, sociales, familiares, económicas de las adolescentes, Carepa, Colombia. Rev CES Salud Pública. 2017; 8 (1): 25-33.

14. Baeza B, Póo A, Vásquez O, Muñoz S, Vallejos C. Identificación de Factores de Riesgo y Factores Protectores del Embarazo en Adolescentes de la Novena Región. Rev. chil. obstet. ginecol. [Internet]. 2007 [citado 2019 Nov 20]

72( 2 ): 76-81. Disponible en: https://scielo. conicyt.cl/scielo.php?script=sci_arttext\&pi$\mathrm{d}=$ S0717-75262007000200002\&lng=es. http:// dx.doi.org/10.4067/S0717-75262007000200002.

15. Mendoza Tascón L, Claros Benítez D, Peñaranda Ospina C. Actividad sexual temprana y embarazo en la adolescencia: estado del arte. Rev. chil. obstet. ginecol. [Internet]. 2016 Jun [citado 2019 Nov 28] ; 81( 3 ): 243-253. Disponible en: https:// scielo.conicyt.cl/scielo.php?script=sci_arttext\&pi$\mathrm{d}=$ S0717-75262016000300012\&Ing=es. http:// dx.doi.org/10.4067/S0717-75262016000300012.

16. Svanemyr J, Guijarro S, Butron B, Chandra-Mouli, V. The health status of adolescents in Ecuador and the country's response to the need for differentiated healthcare for adolescents. Reprod Health. 2017 ; 14: 29. doi: 10.1186/s12978-017-0294-5

17. Sezgin AU, Punamäki RL. Impacts of early marriage and adolescent pregnancy on mental and somatic health: the role of partner violence. Arch Womens Ment Health. 2019. https://doi. org/10.1007/s00737-019-00960-w

18. Baeza B, Póo A, Vásquez $O$, Muñoz S, Vallejos C. Identificación de Factores de Riesgo y Factores Protectores del Embarazo en Adolescentes de la Novena Región. Rev. chil. obstet. ginecol. [Internet]. 2007 [citado 2019 Nov
20] ;72( 2 ): 76-81. Disponible en: https://scielo.conicyt.cl/scielo.php?script=sci_arttext\&pi$d=S 0717-75262007000200002 \&$ Ing =es. http:// dx.doi.org/10.4067/S0717-75262007000200002.

19. Cercado-Mancero A, Fariño-Cortez J, Vera-Lorenti F, Maridueña-Silva $H$, Pacheco-Vila $L$, Real-Cotto J. Factores psicosociales que influyen en el embarazo en adolescentes de un colegio de la provincia de Guayas. Revista científica digital INSPILIP V. 2018; 2 (2). Disponible en https://www. inspilip.gob.ec/

20. Mendoza Tascón L, Claros Benítez D, Peñaranda Ospina C. Actividad sexual temprana y embarazo en la adolescencia: estado del arte. Rev. chil. obstet. ginecol. [Internet]. 2016 Jun [citado 2019 Nov 28] ; 81( 3 ): 243-253. Disponible en: https:// scielo.conicyt.cl/scielo.php?script=sci_arttext\&pi$d=S 0717-75262016000300012 \& \operatorname{lng}=$ es. http:// dx.doi.org/10.4067/S0717-75262016000300012.

21. Encuesta Nacional sobre Relaciones Familiares y Violencia de Género contra las Mujeres - ENVIGMU (2019) disponible en la web: https://www. ecuadorencifras.gob.ec/documentos/web-inec/ Estadisticas_Sociales/Violencia_de_genero_2019/ Boletin_Tecnico_ENVIGMU.pdf

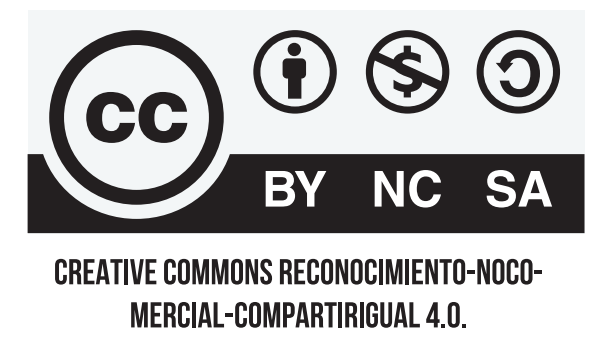

\section{CITAR ESTE ARTICULO:}

Maldonado Rengel, R. E., Suarez, R., \& Rojas Rodríguez, A. L. (2020). Una mirada distinta al embarazo adolescente. RECIMUNDO, 4(4), 228-235. https://doi.org/10.26820/recimundo/4.(4).noviembre.2020.228-235 